\title{
Sec and Tat collaborate in a Rieske business
}

\section{$(c 4$}

polytopic membrane

proteins

are initially

inserted into

the membrane

by the Sec

machinery but

can disengage

and use the

Tat machinery
Protein transport across, or insertion into, the bacterial cytoplasmic membrane occurs through one of a number of proteinaceous channels, or translocons. In the general secretory (Sec) pathway, unfolded proteins are targeted in either a post-translational or co-translational manner to the SecYEG-YidC machinery for translocation or insertion. By contrast, the twin-arginine translocation (Tat) pathway relies on TatABC to translocate or insert proteins that have been fully folded in the cytoplasm, often around a cofactor. Writing in the Journal of Cell Biology, Keller et al. now identify a polytopic membrane protein that requires the action of both the Sec and Tat pathways to ensure correct insertion into the cytoplasmic membrane.

Rieske iron-sulphur proteins are essential components of the bacterial cytochrome $b c_{1}$ and cytochrome $b_{6} f$ complexes and have previously been shown to be Tat substrates. Interestingly, whereas most Rieske proteins have only a single amino-terminal transmembrane (TM) domain, those found in Gram-positive actinobacteria such as Streptomyces spp. are longer and are predicted to contain three TM domains, and the twin-arginine motif used to target the protein to the Tat pathway is located adjacent to the third domain.

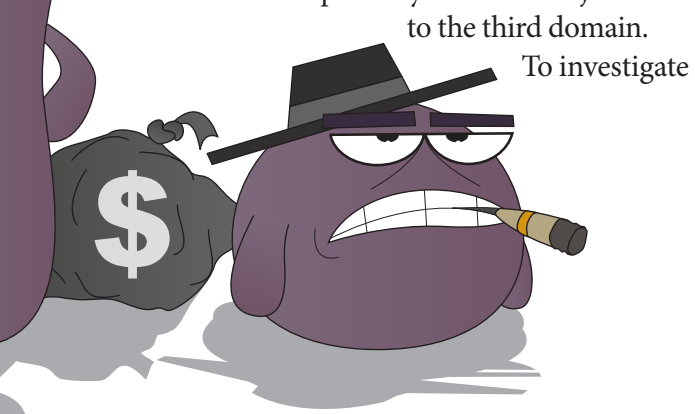

the features of the Streptomyces coelicolor Rieske protein (Sco2149) that are required for its membrane insertion, Keller et al. created two fusion proteins in which the globular iron-sulphur domain of Sco 2149 was replaced with either the mature domain of maltose-binding periplasmic protein (forming TM123-Mbp) or the mature region of AmiA (forming TM123-AmiA), both of which can act as reporters for correct translocation of the fusion protein carboxyl terminus into the periplasm. As expected, they found that when expressed in wild-type Escherichia coli, both proteins were inserted into the membrane with the reporter domains oriented into the periplasm, whereas when expressed in E. coli lacking the Tat system, or when the twin-arginine motif of the reporters had been substituted for a twin-lysine motif (KK), the reporter domains remained cytoplasmic, consistent with Sco2149 being a canonical Tat substrate. However, during protease digestion assays of cell spheroplasts expressing TM123-Mbp, the authors observed an $\sim 49 \mathrm{kDa}$ cleavage product that was present even in spheroplasts derived from cells lacking the Tat system or expressing the KK mutant, suggesting that some portion of the fusion protein was accessible to the protease at the periplasmic face of the membrane, probably the loop between TM1 and TM2. Consistent with this idea, the authors found that TM123-Mbp and TM123-AmiA could not be chemically extracted from crude membrane preparations from cells lacking the Tat system, suggesting that these reporters were indeed integrated into the membrane.
To investigate whether the SecYEG-YidC machinery had a role in the insertion of these fusion proteins, the authors carried out in vitro translation-membrane insertion assays using TM123-AmiA and purified inner-membrane vesicles (IMVs) containing variable levels of SecYEG and YidC. In agreement with insertion by the Sec machinery, they observed an increase in the insertion of both TM123-AmiA and the KK-substituted mutant using IMVs containing elevated levels of either SecYEG or YidC, but a decrease in insertion into IMVs in which YidC had been depleted. Finally, the authors replaced TM1 and TM2 of TM123-AmiA with either two or four TM domains from another E. coli Sec substrate, MtlA, and found that the resulting fusion proteins also required both the Sec and Tat systems for correct insertion into the membrane, indicating that this dual-pathway dependence was not an intrinsic feature of TM1 and TM2 of Sco2149.

The authors propose a model in which polytopic membrane proteins are initially inserted into the membrane by the Sec machinery but can disengage and use the Tat machinery should they contain a globular domain that needs to be translocated in a folded state and would therefore be too wide for the narrow channel formed by SecYEG.

Andrew Jermy

ORIGINAL RESEARCH PAPER Keller, R. et al. Co-operation between different targeting pathways during integration of a membrane protein.J. Cell Biol. 199, 303-315 (2012)

FURTHER READING Palmer, T. \& Berks, B. C. The twin-arginine translocation (Tat) protein export pathway. Nature Rev. Microbiol. 10, 483-496 (2012) 